\title{
EARLY SCIENTIFIC RESULTS AND FUTURE PROSPECTS FOR THE REJUVENATED HUBBLE SPACE TELESCOPE
}

\author{
MALCOLM B. NIEDNER. \\ Laboratory for Exoplanets and Stellar Astrophysics, NASA Goddard Space Flight Center. \\ Greenbelt, MD 20771 , USA \\ E-mail: Malcolm.B.Niedneronasa.gov \\ www.nasa.gov/centers/goddard
}

Following the extraordinarily successful Servicing Mission 4 (SM4) of Hubble Space Telescope (HST) in May of 2009, the Observatory is now fully equipped with a broad array of powerful science instruments that put it at the pinnacle of its scientific power. Relevant to the subject matter of the Beyond 2010 Conference, HST will be well-placed over the next five plus years to advance our knowledge of the formation of high-redshift galaxies and their growth with cosmic time; the emergence of structure in the early universe via Dark Matter-driven gravitational instability; and the universe's expansion history and any resulting implications for the temporal character of Dark Energy. These are fitting projects for the iconic facility now celebrating its 20 th anniversary in orbit.

Keywords: Universal expansion, Dark Energy, Galaxy formation, Dark Matter.

\section{The Goals of Hubble Servicing Mission 4}

With an originally planned science lifetime of fifteen years (1990-2005), Hubble Space Telescope (HST) is the one space observatory that from the outset was designed to be serviced by astronauts. The goals of servicing have always been two-fold: to sustain and extend science operations by replacing/upgrading key components of the engineering infrastructure that supports and enables that science, and to install successively more powerful scientific instruments as technology rapidly advances on the ground. It is beyond dispute that servicing has been the key to Hubble's unique success as an orbiting observatory capable of a continuing wide and diverse range of critical inquiry.

It is highly probable--and it is NASA's formal goal- that HST's science life has been extended to at least 2014 (and perhaps several years beyond), the enabling event being the unprecedentedly complex and successful Servicing Mission 4 (SM4) in May, 2009. This daunting mission accomplished everything possible in a full fivespacewalk flight to address both the engineering and scientific needs of the telescope. Concerning the former, full sets of six gyroscopes and six batteries were installed, as well as a fine guidance sensor, a science instrument controller, and three panels of thermally insulating material for several of the key electronics bays.

Before describing the early new science emerging from the rejuvenated Hubble 
(with some looking back when appropriate, in commemoration of Hubble's 20th anniversary), I will give brief overviews of the scientific objectives of SM4, specifically the installation of two new instruments-WFC3 and COS-and the successful repair of two existing ones-ACS and STIS. The full set of mission objectives, the history leading up to SM4, and the mission itself, are described by Niedner. ${ }^{1}$

\subsection{Wide Field Camera 3 (new)}

Wide Field Camera 3 (WFC3) was built as a "facility instrument" (no Principal Investigator) to guarantee Hubble's high-resolution, wide-field imaging - its signature product - to the end of the science mission. An additional, critical objective was that WFC3 have the capabilities to go beyond the performance of previous Hubble wide-field imagers (Wide Field and Planetary Camera 2, Advanced Camera for Surveys) by offering first-ever panchromatic coverage-from the ultraviolet (UV) through the near-infrared (200-1700 $\mathrm{nm}$ )-over a wide field and with high sensitivity. The panchromatic performance is shown in the right panel of Figure 1. The heart of the instrument is the detectors in the two science channels: two butted $2 \mathrm{k} \times 4 \mathrm{k}$ pixel ( $160 \times 160$ arcsecond field of view) UV/blue-optimized CCDs in the "UVIS channel," which is sensitive over the range 200-1000 $\mathrm{mm}$; and a $\mathrm{Hg}-\mathrm{Cd}-\mathrm{Te}$ $1 \mathrm{k} \times 1 \mathrm{k}$ pixel $(123 \times 135 \mathrm{arcsec})$ detector in the "IR channel," with a spectral sensitivity range of 800-1700 $\mathrm{nm}$. Both detectors are state-of-the-art devices and are the end result of many years of development and steady improvement. They provide substantially more than order-of-magnitude gains over past HST instruments in key performance areas. WFC3's other key advantage over previous imagers is its large and diverse set of spectral elements: 47 filters and 1 grism in the UVIS channel, and 15 filters and 2 grisms in the IR. A more complete technical description WFC3 is given by Kimble et $a l^{2}$ and MacKenty et al. ${ }^{3}$ Instrument details are also given

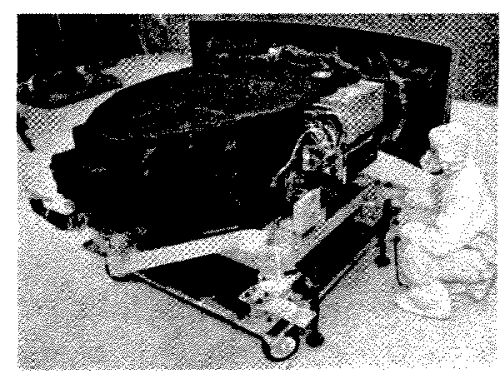

(a)

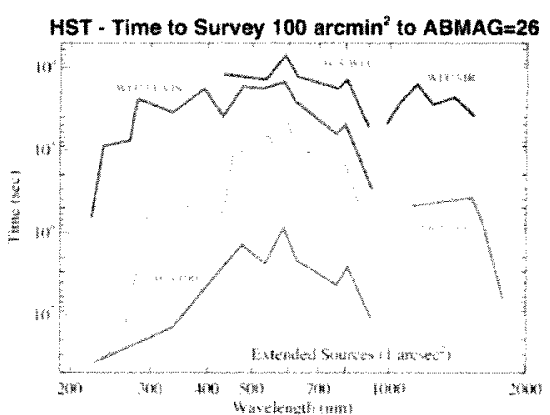

(b)

Fig. 1. (a) the fully integrated Wide Field Camera 3 (WFC3) in the large clean room at NASAGoddard Space Flight Center. (b) survey speeds of the two WFC3 channels vs. ACS, NICMOS and WFPC2 (removed from HST in SM4). WFC3 brings unique performance in the near-UV/blue and the near-1R. ${ }^{4}$ 
in the WFC3 Instrument Handbook maintained by the Space Telescope Science Institute. ${ }^{4}$

\subsection{Cosmic Origins Spectrograph (new)}

Prior to SM4, HST spectroscopy had evolved spectacularly from the first-generation Goddard High Resolution Spectrograph (GHRS) and Faint Object Spectrograph (FOS), to the ultra-versatile, and in some ways still unsurpassed, second-generation Space Telescope Imaging Spectrograph (STIS).

Even given all its available observing modes, however, one thing was missing from STIS: optimized sensitivity in the UV, particularly the far-UV (FUV). Cosmic Origins Spectrograph (COS) was proposed by Principal Investigator James Green to enhance that missing performance by a factor of at least $10 x$, and the solution was a simple, elegant design in which light entering COS experiences one optical bounce prior to entering the FUV detector; cf. Green et al. ${ }^{5}$ and Froning and Green. ${ }^{6}$ The bounce is off of one of four selectable diffraction gratings that, in addition to dispersing the light, correct for HST's spherical aberration as well as local astigmatism. The FUV channel nominally covers the spectral range 115-175 $\mathrm{nm}$, but has some useful sensitivity below $100 \mathrm{~nm}$. Its detector is a photon-counting cross delay line device with a CsI photocathode.

The second COS channel covers the near-UV (175-320 nm) with four gratings and a CsTe photocathode Multi-Anode Multichannel Array (MAMA). Although optically less simple than the FUV channel, COS's NUV performance is still faster by $3-4 \mathrm{x}$ than that of STIS. Details on COS are given in Refs. 5 and 6 and the COS Instrument Handbook ${ }^{7}$

\subsection{Advanced Camera for Surveys (repaired)}

Advanced Camera for Surveys (ACS) was installed on HST during SM3B in March, 2002, and is its widest field-of-view imager. Its Wide Field Channel (WFC) projects a $202 \times 202$ arcsecond field onto two butted $2 \mathrm{k} \times 4 \mathrm{k}$ CCDs that have sensitivity over the range $380-1000 \mathrm{~nm}$. As ACS was designed primarily for cosmology and lacked a separate "IR channel," the WFC CCD was red-optimized and has maximum response at $\sim 600 \mathrm{~nm}$ (Ford et al. ${ }^{8}$ ). ACS is probably most well known for the Hubble Ultra Deep Field of 2004 (Beckwith et al. $^{9}$ ), as well as for wide-field cosmological surveys such as "GOODS" (Giavalisco et al. ${ }^{10}$ ) and "COSMOS" (Scoville et al. ${ }^{1,12}$ ).

The successful SM4 repair effort for ACS was devoted to the WFC, which during 2002-2007 accounted for $\sim 70 \%$ of ACS science and hence was the instrument's scientific backbone. ACS's other CCD channel, the narrow-field High Resolution Channel (HRC), was not repaired during SM4. Historically HRC had accounted for $20 \%$ of ACS science. The remaining $10 \%$ was performed by the instrument's Solar Blind Channel (SBC), which has worked continuously from 2002 to the present.

More details about the nature of the 2007 failure that took down WFC and $\mathrm{HRC}$, as well as the SM4 repair technique that restored WFC, are reported in 
Refs. 1 and 13. It is important, to note that with their different unique features, as well as important areas of overlap, WFC3 and ACS complement and back each other up beautifully. ${ }^{4,14}$

\subsection{Space Telescope Imaging Spectrograph (repaired)}

Space Telescope Imaging Spectrograph (STIS) was installed on HST during SM2 in February, 1997. With an array of long slits of different width to choose from, the availability of low-medium-high spectral resolution, extremely wide UV-Visible-NIR spectral coverage (121-1100 nm) offered by two UV MAMA detectors (FUV/CsI and NUV/CsTe photocathodes) and a CCD (200-1100 nm), STIS offered the observer powerful diffraction-limited 2-d spectroscopy and arguably became HST's most versatile instrument (my judgment; cf. Woodgate et al. ${ }^{15}$ and Kimble et al. ${ }^{16}$ for instrument details). Its two signature achievements (pre-SM4) were almost certainly: 1.) the efficiency with which it mapped the velocity fields of galaxies and detected supermassive black holes (SMBHs) in their nuclei, leading to the view that most, if not all galaxies possess SMBHs that have a role in their formation and/or evolution; ${ }^{17,18}$ and 2.) the highly unanticipated commencement of the study of exoplanetary atmospheres in transiting star-planet systems. ${ }^{19.20}$

The failure of a $5 \mathrm{~V}$ power converter took STIS down in August, 2004. Because of its uniqueness and very high degree of complementarity with the (then) future COS, planning for the SM4 repair of STIS began almost immediately, and by late2005 was well underway. For more about the details of the 2004 STIS failure and the techniques that successfully restored it during SM4, refer to Refs. 1 and 21 . In addition to Refs. 15 and 16, a full description of STIS is found in the Instrument Handbook. ${ }^{22}$

\subsection{Near Infrared Camera and Multi-Object Spectrometer (untouched in SM4)}

This fifth HST instrument-which in the most recent observing cycle was responsible for $\sim 2 \%$ of the total HST observing time--was not worked on or touched by SM4 astronauts. At the current time the mechanical cryocooler that cools the IR detectors to $\sim 77 \mathrm{~K}$ is not rumning due (apparently) to trace amounts of water ice which have migrated to problematic areas in the Neon circulator loop that cools the instrument. HST Program has developed a technique to flush the loop of its current $\mathrm{Ne}-\mathrm{H}_{2} \mathrm{O}$-other content and refill it with pure $\mathrm{Ne}$ from the on-board refill tank. Before WFC3 was installed and largely subsumed its science, NICMOS was a highly used, critical asset on HST, its most notable achievements being: the precise photometry of high-redshift Type Ia supernovae vital for HST Dark Energy work (cf. Riess et al. ${ }^{23.24}$ ); and its important contribution to probing the molecular content of exoplanetary atmospheres (Swain et al. ${ }^{25}$ ). 


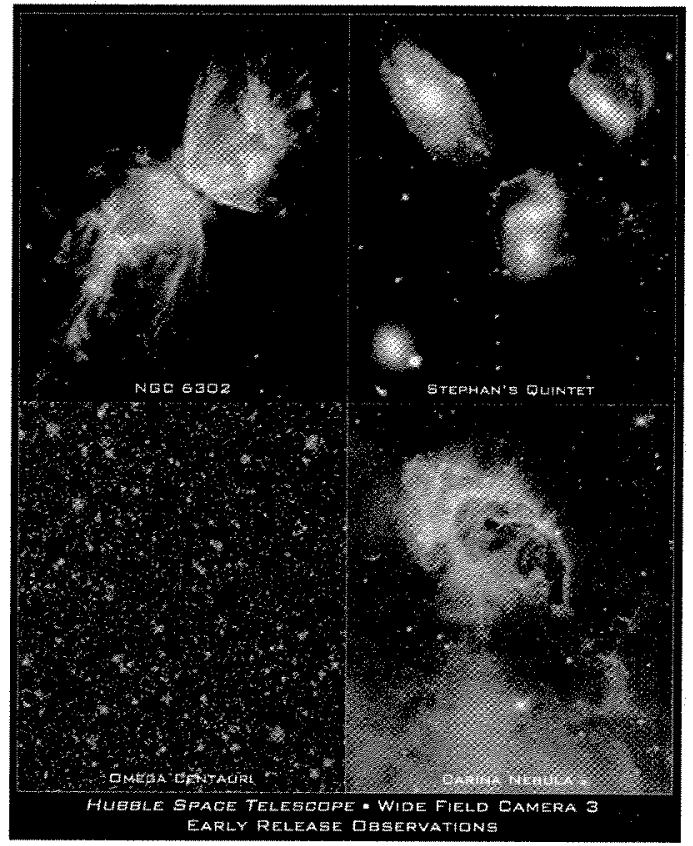

Fig. 2. The Early Release Observations (EROs) produced by WFC3, released on September 9, 2009.

\section{Early Post-SM4 Science and Prospects, with a Cosmological Bent}

Although a year has elapsed since SM4 at the time of this writing, the rejuvenated Hubble is still quite young and observers have not had much time to analyze and publish their data since the multi-month Servicing Mission Observatory Verification (SMOV) activities completed in the fall of 2009. Even so, refereed published papers are beginning to appear, and it is quite clear that HST is significantly more powerful than it ever has been. For the remainder of the paper I will concentrate on three subject areas:

(1) Refining $\mathrm{H}_{0}$ and constraining Dark Energy

(2) Going the distance: searching for and characterizing the most remote galaxies

(3) Probing Dark Matter distribution through Strong and Weak Lensing

First, however, it is worth reliving the first public WFC3 images to come out of SM4, its "Early Release Observations" (EROs). They are shown in Figure 2.

\subsection{Refining $H_{0}$ and Constraining Dark Energy}

At the risk of oversimplification, I take the view that between the mid-1990s and the present, the Hubble constant, $\mathrm{H}_{0}$, and Dark Energy have had two distinct, 
important, and rather different "encounters" in HST-based research. The second encounter is ongoing.

1994-2001. "Determination of the Extragalactic Distance Scale" was a Key Project (J. Mould, Principal Investigator) at HST's 1990 launch; its goal was the measurement of $\mathrm{H}_{0}$ to an accuracy of $10 \%$, a cosmologically critical task for establishing the age and scale of the universe. A similar program, called the HST Supernova Calibration Program (A. Saha, PI), had the same overall objective.

Pre-HST, measuring $\mathrm{H}_{0}$ was a daunting issue due to the great difficulties in determining accurate galaxy distances. Recession velocities were of course well measured and not a noise term of any significance for $\mathrm{H}_{0}$ determinations. As a result of the distance measurement problem (the same issue that plagued Edwin Hubble), astronomers disagreed about $\mathrm{H}_{0}$ 's value by a factor of $\sim 2 \mathrm{x}\left(\sim 50 \mathrm{~km} \mathrm{~s} \mathrm{~m}^{-1}\right.$ Megaparsec ${ }^{-1}[\mathrm{Mpc}]$ vs. $\sim 100 \mathrm{~km} \mathrm{~s}^{-1} \mathrm{Mpc}^{-1}$; cf. Sandage ${ }^{26}$ for a discussion). The $\mathrm{H}_{0}$ Key and Supernova Calibration Projects aimed to take direct advantage of HST's combination of superb angular resolution and sensitivity-after installation of the Wide Field and Planetary Camera 2 (WFPC2) in 1993 vanquished HST's spherical aberration problem - to perform accurate photometry of distance-yielding Cepheid variable stars over a local volume of galaxies significantly larger than had been previously possible. Although these "Hubble Cepheids" would not by themselves reach far enough to suppress to an acceptably low level the random velocities of galaxies as a fraction of the universal expansion out to the farthest Cepheid distances, they would permit the calibration of much more luminous, far-reaching secondary distance indicators present in the HST-observed "Cepheid galaxies." Thus could be obtained accurate distances out into the true "Hubble How," producing a welldetermined value for $\mathrm{H}_{0}$ (Freedman et al.; ${ }^{27}$ Sandage et al. ${ }^{28}$ ). Among the several secondary distance methods and indicators were Type Ia supernovae (SNe), the most accurate yardstick for distance determination across an appreciable fraction of the observable universe. ${ }^{23,24,27,28}$

Preliminary results from the Key Project produced $\mathrm{H}_{0}=80+17 \mathrm{~km} \mathrm{~s}^{-1} \mathrm{Mpc}^{-1}$ (Freedman et al. ${ }^{29}$ Kennicutt et al. ${ }^{30}$ ), but this raised a profound question. Specifically, bringing the $\mathrm{H}_{0}$-inferred (expansion) age of the universe into line with the estimated ages of the oldest (globular cluster) stars seemed to require all three of the following to be true: ${ }^{29,30}$ 1.) the universe is open, with low matter density, $\Omega_{\text {Total }}$ $=\Omega_{\text {Matter }} \leq 0.3 ; 2$.) $\mathrm{H}_{0}$ 's true value is at the low end of the error-bracketed range (i.e., in the low 60s); and 3.) the ages of the oldest stars are on the low end of their estimated range. In a closed, all-matter Einstein-de Sitter miverse, however, the problem with the Key Project $\mathrm{H}_{0}$ result was profound: the expansion age and oldest stellar ages disagreed by $\sim 6$ Gyr (universe younger ${ }^{29.30}$ ). As Freedman et al. and Kennicutt et al. stated, a possible way out of the dilemma was through the agency of a non-zero cosmological constant $(\Lambda)$, but no data directly supporting this scenario were presented.

The Saha et al. team, in the meantime, had derived a preliminary value of $H_{0}$ $=58+7 \mathrm{~km} \mathrm{~s}^{-1} \mathrm{Mpc}^{-1,31}$ and what was noteworthy was that using the same 
methods and many of the same targets, the two teams using HST were deriving values of $\mathrm{H}_{0}$ that were still in disagreement at the $25 \%$ level.

The Key Project's final result ${ }^{27}$ was $\mathrm{H}_{0}=72+/-8 \mathrm{~km} \mathrm{~s}^{-1} \mathrm{Mpc}^{-1}$, and that of the Supernova Calibration Project ${ }^{28}$ was $\mathrm{H}_{0}=62.3+/-5.2 \mathrm{~km} \mathrm{~s}^{-1} \mathrm{Mpc}^{-1}$. The Key Project, in particular, would have simply confirmed and reinforced the justcited age dilemma had not new results already emerged on $\mathrm{H}_{0}$ 's time variability that radically changed our understanding of the universe's expansion history, and in the process resolved the expansion age vs. stellar age conflict that otherwise existed for an $\mathrm{H}_{0}$ in the low $70 \mathrm{~s}$.

Making use of Type Ia SNe observations to pin down the value of the cosmological "deceleration parameter," Riess et al. ${ }^{32}$ and Perlmutter et al. ${ }^{33}$ had foundmostly on the strength of ground-based data, but also with a few higher redshift, greater accuracy, and hence high-value HST observations - that the local universe is not decelerating at all, but accelerating (by "local" is meant out to several Gyr lookback time). Acceleration, if it survived the test of future observations, meant that Einstein's famous $\Lambda$ was "back in play," and that locally at least, gravity was being defeated by an unknown dominant repulsive force in the vacuum. ${ }^{32,33}$ Not knowing whether the repulsive force was constant in cosmic time (i.e., whether it actually was $\Lambda$ ) - let alone any of the physics behind it-it was simply named "Dark Energy" (DE). Later HST observations of high-redshift Type Ia SNe provided strong confirming evidence of a currently accelerating universe at the level of $\Omega_{\Lambda} \sim 0.7$ (e.g., Riess et al. ${ }^{34}$ and Knop et al. ${ }^{35}$ ).

To sum up the first "encounter" between $\mathrm{H}_{0}$ and DE in the HST experience, the vexing combination of Kennicutt et al. $\mathrm{s}^{30} \mathrm{H}_{0}$ (consistent with the later Freedman et al. result ${ }^{27}$ ) and the obvious insistence that the oldest stars be no older than the universe was, in a sense, "saved" by the discovery of Dark Energy: a flat universe $\left(\Omega_{\text {total }}=1\right)$ with $\mathrm{H}_{0} \sim 72 \mathrm{~km} \mathrm{~s}^{-1} \mathrm{Mpc}^{-1}$ and $\Omega_{\Lambda} \sim 0.7$ placed the universe and oldest stars in the same age box of $14+/-1$ Gyr. The age conflict was resolved, but it came at the expense of a profound puzzle for physics and astrophysics (a joyful gift to some?): what exactly is Dark Energy?

2009-2010- The second HST $H_{0}$-DE encounter is ongoing, and it represents the very different, and in some sense "reversed" interaction (at least to this author), in which further refinements of $\mathrm{H}_{0}$ have strong implications for DE. A team led by Adam Riess is undertaking this effort, called "Supernovae and $\mathrm{H}_{0}$ for the Equation of State" (or $\mathrm{SH}_{0} \mathrm{ES}$ ), and the remainder of this section will briefly discuss recently obtained results, the factors that made them possible, and the prospects for further advances using the post-SM4 HST.

Given WMAP's 5-year measurement of the product $\Omega_{\text {Matter }} \mathrm{H}_{0}^{2}$ to $5 \%$ accuracy (Komatsu et al ${ }^{36}$ ), a significant reduction of Freedman et al.'s ${ }^{27} 10-11 \%$ uncertainty in $\mathrm{H}_{0}$ to $\leq 5 \%$ can by itself substantially constrain the uncertainty in the Dark Energy equation of state, $w=P /\left(\rho c^{2}\right)$, and specifically test whether $A$ is supported by a finding of $w=-1$ to within the errors (Riess et al. ${ }^{24}$ ). To jump to the conclusions, Riess et al.'s $\mathrm{SH}_{0}$ ES Team improved our knowledge of $\mathrm{H}_{0}$ to $74.2+$ 


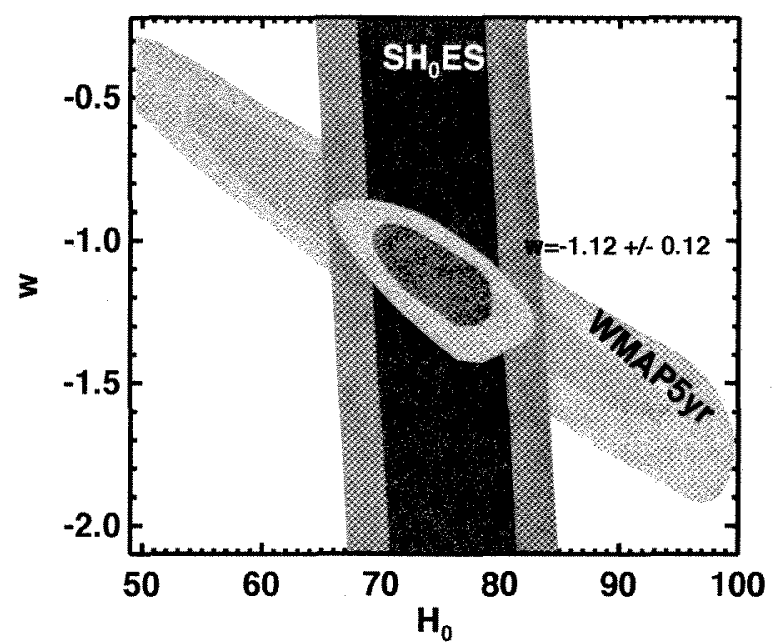

Fig. 3. Probability contour diagram from Riess et al. ${ }^{24}$ showing the intersection of WMAP 5year results and those of the $\mathrm{SH}_{0} \mathrm{ES}$ program in the $w$ - $\mathrm{H}_{0}$ plane. $\mathrm{SH}_{0} \mathrm{ES}$ result of $\mathrm{H}_{0}=74.2+/-$ $3.6 \mathrm{~km} \mathrm{~s}^{-1} \mathrm{Mpc}^{-1}$ produces a most likely equation of state parameter $w$ which is consistent with -1 and the cosmological constant $\Lambda$. The innermost contour denotes a confidence level of $68 \% .24$

$3.6 \mathrm{~km} \mathrm{~s}^{-1} \mathrm{Mpc}^{-1}$ (4.8\%), and with the WMAP 5-year results and the assumption of a constant equation of state $w$ and a flat universe, they derived $w=-1.12+0.12$ (refer to Figure 3), which is both consistent with a cosmological constant and has smaller error bars (by half) than other data combinations such as WMAP + Baryon Acoustic Oscillations (BAO) and WMAP + Freedman et al.'s $\mathrm{H}_{0}$ (cf. Ref. 24). Note that the $\mathrm{SH}_{0} \mathrm{ES}$ result agrees with Freedman et al.'s $\mathrm{s}^{27} \mathrm{H}_{0}$ measurement.

Removing the constraints of a flat universe with constant $w$, and utilizing BAO data, WMAP 5-year results, and high-redshift (z) SNe, Riess et al. ${ }^{24}$ found that their $2.2 \mathrm{x}$ reduction in $\mathrm{H}_{0}$ uncertainty led to a run of $w(\mathrm{z})$ that in three separate redshift bins out to $\mathrm{z}=1.8$ were all consistent with $\Lambda$, showing no evolution with $\mathrm{z}$ to within the errors. As a further demonstration of the importance of increasing accuracy in $\mathrm{H}_{0}$, Riess et al. determined that the Dark Energy "figure of merit" (FoM)--in this instance the inverse product of the uncertainties of $w$ in the three redshift bins-increased three-fold compared to when the Freedman et al. value of $\mathrm{H}_{0}$ and its uncertainty were used. The message: highly accurate knowledge of $\mathrm{H}_{0}$ matters.

It is important to describe how HST and the $\mathrm{SH}_{0} \mathrm{ES}$ program produced such an improved result for $\mathrm{H}_{0}$, becanse it illustrates the enormous improvements that have been made in Hubble through successive servicing missions, as well as improvements in observational strategies and opportunities. Furthermore, $\mathrm{SH}_{0} \mathrm{ES}$ is broadly indicative of the future progress that HST will make in the study of Dark Energy. 
Freedman et al. ${ }^{27}$ observed their Cepheids with the Wide Field and Planetary Camera 2 (WFPC2), the iconic instrument that, upon its installation on HST in December 1993 during SM1, instantly vanquished spherical aberration and brought diffraction-limited wide-field imaging to Hubble for the first time (Holtzman et $a l^{37}$ ). Using WFPC2 and Cepheids, Freedman et al. derived galaxy distances and calibrated the secondary distance standards within them, such as Type Ia SNe, using Large Magellanic Cloud (LMC) Cepheids as their "anchor" to put the distances on an absolute scale. As Freedman and her colleagues noted, the $8 \mathrm{~km} \mathrm{~s}^{-1}$ error bars on $\mathrm{H}_{0}$ were driven, in part, by WFPC2 instrumental issues such as error in the photometric zero point. Uncertainty in the distance to the "anchor," the LMC, also was a substantial error term. ${ }^{24,27}$

Advanced Camera for Surveys (ACS) was installed on HST in March, 2002 during SM3B. It offered enormous advantages over WFPC2: improved photometric properties, higher sensitivity, and twice the angular resolution and areal field of view. By extending to $\sim 30-35 \mathrm{Mpc}$ the distance at which Cepheids are resolved and measurable, vs. $\sim 20 \mathrm{Mpc}$ for WFPC2, ${ }^{23,24}$ a much greater statistical sample of Cepheids was available and the probability that a new (or recently measured) Type Ia supernova would occur (had occurred) within Hubble's "Cepheid radius" was much increased. Those were some of the differences in instrumental factors between Freedman et al.'s and Riess et al.'s $\mathrm{H}_{0}$ determinations, and they strongly favored Riess et al.'s use of ACS. Refer to Figure 4.

There is more to the story than the use of ACS, however. While $\mathrm{SH}_{0} \mathrm{ES}$ has taken
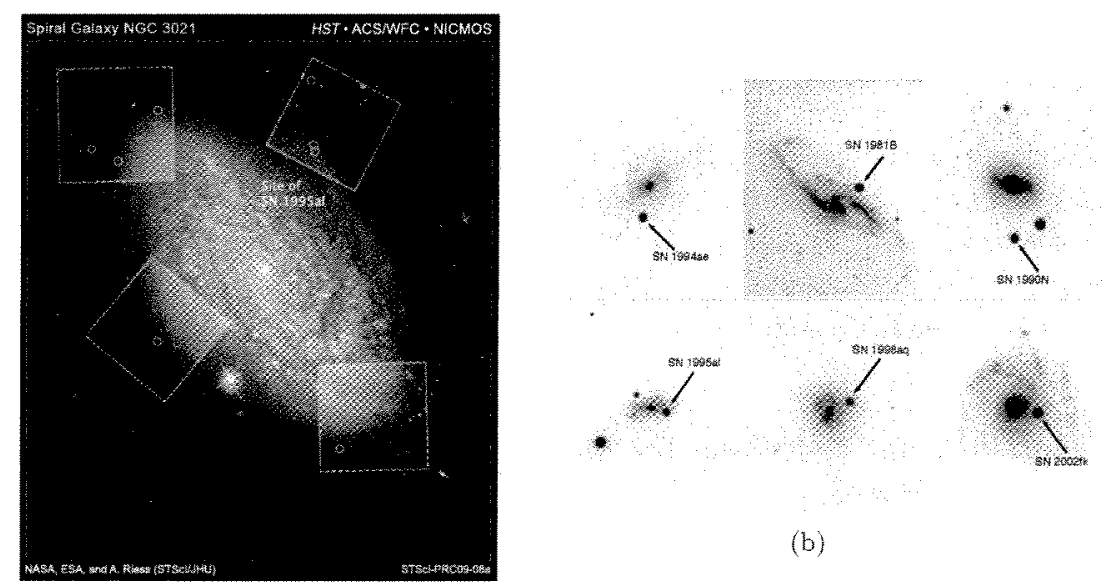

(b)

(a)

Fig. 4. (a) ACS frame of NGC 3021, a spiral galaxy supernova host. Cepheids (circled) were detected by ACS, with follow-up observations by NICMOS, whose field of view is indicated by the squares; from Refs. 23 and 24 . (b) ground-based images showing six galactic hosts of "high-quality" Type Ia supernovae of particular interest to the $\mathrm{SH}_{0} \mathrm{ES}$ team. ${ }^{23,24}$ Note that $\mathrm{SN} 1995 \mathrm{al}$ (lower left) occurred in NGC 3021 (left frame). 
full advantage of ACS to detect and accurately characterize Cepheids to greater distances in an efficient manner, it has also utilized the Near-Infrared Camera and Multi-Object Spectrometer (NICMOS) to suppress errors due to dust extinction of light and chemical variations among Cepheids, the seriousness of both of which is strongly reduced in the near-IR as compared to the visible (cf. Refs. 23 and 24 for particulars).

One final detail in $\mathrm{SH}_{0} \mathrm{ES}$ ' "tools and methods" should be mentioned: the choice of distance anchor. Riess et al. chose the maser host, accretion galaxy NGC 4258, as their anchor, the advantage of which was the unprecedented (for an extragalactic object) $3 \%$ accuracy of its measured distance. This derives from the fact that the Doppler-shifted water maser clouds revolving around the central black hole (BH) have very well measured velocities, proper motions, and angular distances from the $\mathrm{BH}$; hence the distance calculation is one of those rare cases in which geometry is all that is required (Argon et al. ${ }^{38}$ ). Moreover, NGC 4258 is close enough at $7.2 \mathrm{Mpc}$ that its Cepheids are easily observed, and in great numbers. In contrast, Freedman et $a .^{27}$ used the LMC as their anchor, the distance of which was known only to within $5 \% .^{24,27}$

As Riess et al. ${ }^{24}$ argue, further progress can be made in the accuracy of $\mathrm{H}_{0}$, and the $\mathrm{SH}_{0} \mathrm{ES}$ Team is currently using the higher sensitivity, larger field, and superior photometric properties of HST's WFC3/IR channel, to improve the earlier measurements made with NICMOS. The goal is to reduce the error on $\mathrm{H}_{0}$ from $4.8 \%$ to $\leq 3 \%$. It is expected that in future years additional well-measured maser systems will become available, eliminating the current reliance on one anchor and reducing the errors via averaging. Establishing absolute distances of multiple maser systems is a goal of the Megamaser Cosmology Project. ${ }^{39,40}$ The $\mathrm{SH}_{0} \mathrm{ES}$ Team has shown through simulation that an $\mathrm{H}_{0}$ known to $1 \%$ accuracy (a lofty but perhaps achievable future goal), when used with data from aggressive Type Ia SNe and BAO observing programs, can increase the Dark Energy FoM enormously. Theoretical understanding of $\mathrm{DE}$ will almost certainly depend on progress being made toward a much better observational characterization of it than exists today, and HST will surely be a player in these future studies. The reader is referred to Frieman et al. ${ }^{41}$ for a current review of Dark Energy research.

\subsection{Going the Distance: Searching for and Characterizing the Most Remote Galaxies}

Though not among the original goals of the 1977 NASA/HST Announcement of Opportunity, the detection and characterization of ever more distant galaxies has for fifteen years been a high-profile and hugely productive HST effort whose motivation has, in part, been tied to the need to understand the population of local galaxies (those in the "here and now," such as the Milky Way and Andromeda galaxies) as the end products of galaxy formation and evolution from the earliest of cosmic times. 
Other reasons exist for the search of the earliest galactic systems, among them understanding the process(es) by which the so-called "Dark Ages"-_-during which the intergalactic medium (IGM) consisted of pure neutral species dominated by $\mathrm{H}$ and $\mathrm{He}$--ended via the Dark Matter-driven emergence of the first stellar systems and perhaps accreting black holes (BH) (Fan et al.; $;^{42}$ Loeb; $;{ }^{43}$ White and Rees ${ }^{44}$ ). FUV photons from either or both the fledgling galaxies and black holes would have ionized the IGM, if copious enough. Among the questions are: were galaxies alone sufficient, or were BHs also needed, and what were their fractional contributions to reionization?

Understanding the physical details, the central epoch, and the cosmic time interval over which this last major change of state - the reionization of the universeoccurred is a major focus of astrophysics today. ${ }^{42,43}$ The post-SM4 HST is more capable than ever to address these questions and will help lead the way for the James Webb Space Telescope (JWST; Gardner $e t$ al. ${ }^{45}$ ) to probe even deeper toward the Big Bang as result of its substantially larger mirror and operation at longer IR wavelengths.

The original effort to go as deep as possible with HST was made with WFPC2 (Williams et $a l^{46}$ ). For ten consecutive days HST and WFPC2 were pointed at an apparently blank piece of sky, and the summed exposures through various filters produced an iconic image that was anything but blank: the "Hubble Deep Field" (HDF) recorded several thousand remote galaxies, some at redshifts $>5$ (cf. review by Ferguson et al. ${ }^{47}$ ), which, with the later $\Lambda$-Cold Dark Matter $(\Lambda \mathrm{CDM})$ concordance cosmology and $\mathrm{H}_{0}=74.2+3.6 \mathrm{~km} \mathrm{~s}^{-1} \mathrm{Mpc}^{-1},{ }^{24}$ represents light emitted only $\sim 1$ Gyr after the Big Bang. A later "HDF-South" was observed with WFPC2, NICMOS, and STIS, and NICMOS observations were also made of the original HDF-North (cf. Ref. 47 for an HDF review).

A word about redshift determination for these remote, challenging targets is in order. As the most distant galactic objects detected in the Hubble Deep Fields are too faint for direct spectroscopic redshift measurement - even with behemoth ground-based telescopes - the now standard photometric "dropout" technique utilizing filtered imaging and the virtual disappearance of rest-frame flux below 91.2 and $121.5 \mathrm{~nm}$ due to absorption by intra- and inter-galactic hydrogen, is used to calculate "photometric redshifts" of usually good accuracy (cf. Steidel et al ${ }^{48}$ for discussion of the technique, and to the right panel of Figure 5 for an illustration of dropouts in the HUDF09, discussed below).

A major advance beyond the HDFs was made in 2004 by ACS and NICMOS in the Hubble Ultra Deep Field (HUDF04; Beckwith et al. ${ }^{9}$ ). Contained in the single ACS field-of-view, ultra-deep "core sample," also observed at depth with NICMOS, were some 10,000 galaxies, the faintest and reddest of which reached $z \sim 7$ (Bouwens et al. ${ }^{49}$ ) and probed to within $\sim 800 \mathrm{Myr}$ of the Big Bang.

By a variety of techniques and lines of evidence, reionization of the universe is thought to have started at $z>11$ and was essentially complete by $z \sim 6$ (e.g., Ref. 42). This of course means that the first stars and galaxies (or their subunits) 
were assembling at $z>10$ and that the HUDF04, for all it achieved by reaching $z$ $\sim 7$, had not probed any but the tail-end of the reionization interval. Because of the undoubted (but unquantified) role galaxies played in the reionization process, and also because of the need to observe even earlier and younger galactic objects from the viewpoint of galaxy formation theory, there was (there always is) a profound need to go deeper, farther, and earlier.

WFC3's IR channel has already shown that it has the performance capability to do just that, as well as to bridge the HST and JWST eras of observational cosmology. G. Illingworth is the PI of a large HST program to repeat the HUDF04 using WFC3/IR and push to redshifts well beyond $\mathrm{z}=7$; the product is referred to as "HUDF09," and is shown in Figure 5. With its large field, excellent sensitivity, and superior photometric properties as compared with NICMOS (installed on HST in 1997), WFC3/IR has observed the identical ACS/NICMOS HUDF04 field and pushed the limits of galaxy detection well beyond what was achieved by the earlier instruments.

A flood of investigations has been triggered as a result of the non-proprietary nature of Illingworth et al.'s data, and space does not permit doing justice to the full set of published and soon-to-be published papers. Several things about WFC3 are clear, however: 1.) the cosmic distance limit for galaxies has been considerably extended by WFC3/IR; and 2.) the efficiency in detecting galaxies at $z \sim 7$ is enhanced (i.e., is faster) by some 40-50x compared to NICMOS (Oesch et al., ${ }^{50}$ Bouwens et al. ${ }^{51}$ McLure et $a .^{52}$ ). Clearly, WFC3 is everything that had been hoped for.

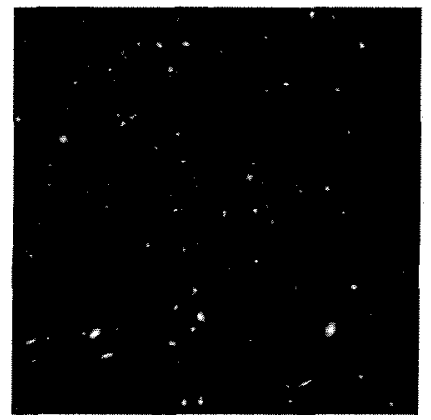

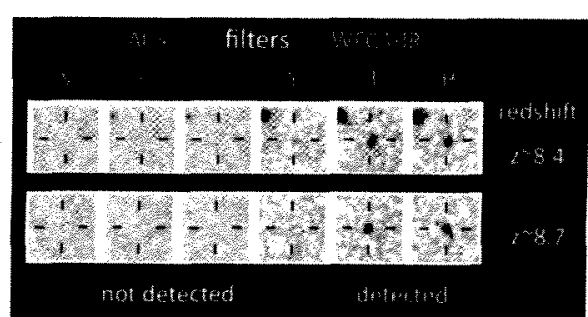

(b)

(a)

Fig. 5. (a) The WFC3/IR summed image comprising the Hubble Ultra Deep Field ("HUDF09"). in which galaxy light as far back as $600 \mathrm{Myr}$ after the Big Bang, and perhaps only $500 \mathrm{Myr}$, is recorded from the most remote galaxies (from Illingworth et al. program). (b) In postagestamp cutouts, HUDF04+09 filter sequences show the photometric "dropout" technique used to derive redshift for two of the highest redshift galaxies. Rest-frame galaxy radiation shortward of the $\mathrm{H}$ ionization edge at $91.2 \mathrm{~nm}$ and the strong Lyman- $\alpha$ line at $121.5 \mathrm{~nm}$ is essentially completely absorbed by intervening $\mathrm{H}$, leading to zero observed flux out to those redshifted wavelengths. Fluxes longward of the dropout are detected and can be modeled to derive a "photometric redshift." From Bouwens et al. ${ }^{5 !}$ 


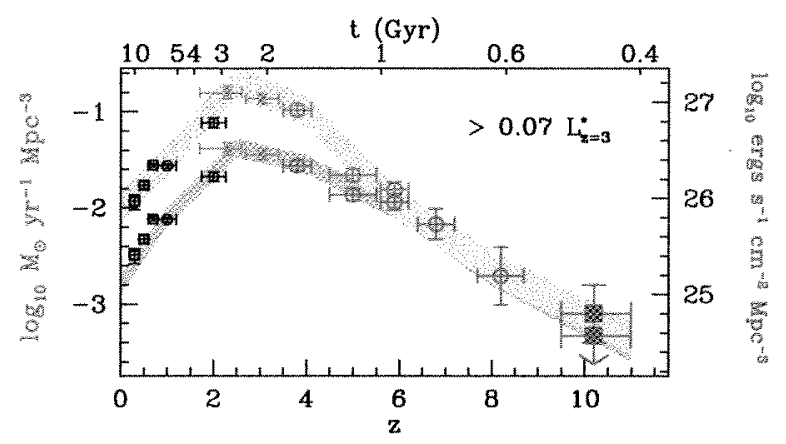

Fig. 6. The HUDF09 extension of galaxy detection to $z \geq 8$ has shown that the earlier trends in galaxy evolution over the range $z=4-7$ continues to $z \geq 8$, and perhaps to $z \sim 10$. Plotted is the observed UV luminosity density in the co-moving frame (right ordinate) and the associated, inferred star formation rate in solar masses per year (left ordinate), as functions of $z$. The lack of vertical error bars out to $z \sim 6$ reflect the fact that the luminosity functions are very welldetermined through high-number statistics, whereas at $z>7$ the fewer numbers of detected objects are placing limits on accuracy. The three highest redshift data points at $z \sim 7$ and 8 , and 10 , are from Refs. 50 and 51 , and Ref. 55, respectively, but the latter is currently pending referee review. Note that the lower blue band contains as-observed galaxies without correction for dust extinction, whereas the upper band contains the dust correction. At high-z the bands overlap due to the absence of dust and "metals." Figure from Ref. 55 .

Not all HUDF-WFC3/IR investigation teams are coming to the same conclusions, but there is apparent agreement that the ease with which the $z=7-8.5$ range has been reached by WFC3 is permitting some conclusions to be drawn about at least the bright end of the galaxy luminosity function (LF) at $600 \mathrm{Myr}$ after the Big Bang, and in particular about the continuation of evolution seen over the lower $z=4-6$ redshift range by WFPC2 and ACS/NICMOS in the HDFs and HUDF04, out to $z=7-8+$. The reader is referred to Refs. 50, 51, and 52, as well as to Fig. 6 .

Specific conclusions in Refs. 50 and 51 are that the $z=7-8.5$ galaxies are extremely small, compact objects with scale size $<1 \mathrm{kpc}$, and that in the rest frame they are extremely blue indicative of a severe deficiency of the heavy element "metals" that are created in successive waves of star formation from the epoch of the first stars to the present. Furthermore, the characteristic galaxy luminosity, "L", decreases monotonically toward higher z (also $\mathrm{cf}$. Ref. 52 ). These results are consistent with the accepted view that hierarchical merging of small galaxies is responsible for the growth of the large systems we see today.

WFC3/IR does not observe to faint enough limits at $z=8-9$ to answer definitely the question of whether galaxy FUV photons are sufficient for reionization. All that can be said at the moment is that extrapolation of the faint end LF slope seen at lower redshifts (assumed to hold at higher redshifts) to $\mathrm{L} / \mathrm{L}^{*} \sim 10^{-4}$ produces an integrated FUV galaxy flux that may be sufficient for all or a majority of that needed for reionization (Trenti et al..$^{53}$ ). The point is that on a relative scale, faint 
galaxies $\left(L \ll L^{*}\right)$ were extremcly numerous and likely produced most of the FUV photons responsible for reionization. ${ }^{52}$

Two last points about the early results coming out of HUDF09 need be made. First, Labbé $e t$ al. ${ }^{54}$ have combined ACS, NICMOS and WFC3/IR data with IR Spitzer data of the same fields. A substantial number of $z=7-8$ objects are seen by Spitzer, which has led to the construction of spectral energy distributions (SEDs) wide enough in wavelength coverage to permit stellar synthesis modeling. The results indicate that these very remote galaxies, seen at $\sim 600$ Myr after the Big Bang, are not young; rather the SEDs point to the existence of stellar populations already $300 \mathrm{Myr}$ old. The second and extremely important point is that $\mathrm{z} \sim 10$ galaxies are claimed to have been detected in submitted HUDF09 papers by Bouwens et al. ${ }^{55}$ and Yan et al., ${ }^{56}$ though the results are very different (Bouwens et al. detecting only 3 objects vs. 20 by Yan et al.).

\subsection{Probing Dark Matter Distribution through Strong and Weak Lensing}

Dark Matter (DM) dominates the mass budget of the universe, and in the concordance $\Lambda$ CMD cosmology its time-dependent distribution drove, and was the major manifestation of, the formation of large-scale structure (cf. Réfrégier ${ }^{57}$ and references contained therein). The use of gravitational lensing as a DM probe on a variety of spatial scales has become widespread and extremely powerful in recent years. A good example is the work by Newman et al.$^{58}$ utilizing HST and the Subaru telescope, in which DM is mapped across three decades of distance from the core of Abell cluster 611 . Lensing has potential uses besides characterizing DM, including the detection of extremely distant galaxies not possible without the high levels of magnification provided by strong lensing of galaxy clusters (Maizy et al. ${ }^{59}$ although cf. Bouwens et al. ${ }^{60}$ for a somewhat different perspective on which portions of the galaxy LF most benefit from strong lensing).

HST has made fundamental contributions in DM research in the strong lensing (SL) enviromments of the inner cores of galaxy clusters (e.g., Smith et al. ${ }^{61}$ and Zitrin et al. ${ }^{62}$ also of. Ref. 58) and in galaxy-galaxy configurations (e.g., Faure et al. ${ }^{63} \mathrm{cf}$. Treu ${ }^{64}$ for a review of SL by galaxies); and in the weak lensing (WL) regime found both in the inner and onter regions of clusters, as well as in the inter-cluster enviromment (discussed in the next paragraph).

Unlike a number of telescopes both on the ground and in space, Hubble is not a survey telescope that maps substantial portions of the sky, but its "wide-field" instruments can, with sufficient tiling, produce deep surveys over fields large enough to beat down cosmic variance. Most notably, the ACS Cosmic Evolution Survey ("COSMOS") produced a medium-deep survey on $1.64 \mathrm{sq}$ deg of contiguous sky that has been extremely powerful for lensing studies, among other things. Massey et al. ${ }^{65}$ for example, performed a 3D cosmic shear (WL) analysis and was able to trace the time-dependent growth of DM clumping and place stronger constraints on 
cosmological parameters than when a more limiting 2D projection technique was used.

It is in the detection of multiply imaged background galaxies - strong lensingthat the diffraction-limited, deep, and multi-waveband imaging of Hubble is perhaps uniquely valuable (e.g., Refs. 61 and 62). ACS has been the heart of such studies since its installation on HST in 2002, but now that WFC3 has become part of the instrument complement, SL work by HST will be more powerful than ever as a result of ACS and WFC3 being able to work efficiently in tandem via the ability to observe simultaneously.

As part of its "Early Release Observation" (ERO) program following SM4, the most distant Abell galaxy cluster was observed by the restored ACS. Abell 370, at a redshift of $z=0.375$, produces through SL what is probably the most well-known of all lensed "giant arcs." A lensed background galaxy at $z=0.725$, the arc was one of the first strong lines of evidence that clusters had sufficiently concentrated DM in their cores to produce strong lensing (Soucail et al.;66,67 cf. also Ref. 68).

Figure 7 shows the ACS observation of A370, as well as a close-up view of the giant arc and several other strongly lensed features. Richard et al. ${ }^{68}$ have analyzed the ACS imagery in detail, and find that the lensed galaxy in the giant arc is a starforming spiral whose nucleus is imaged five times along the arc by the foreground cluster. That the arc is continuous derives from the other, extended structure in the spiral galaxy, including the individual blue star-forming regions. In brief, including the background spiral comprising the giant arc, ACS images of A370 capture 10 separate multiply imaged background galaxies that produce 32 total images. ${ }^{68}$ With such a rich dataset, Richard et al. construct a DM model that, supported by data from the Chandra X-ray Observatory (CXO), demonstrate that Abell 370 is actually two galaxy clusters in collision, their relative velocity vector oriented nearly along the line of sight. HST has detected several other clusters in collision as evidenced

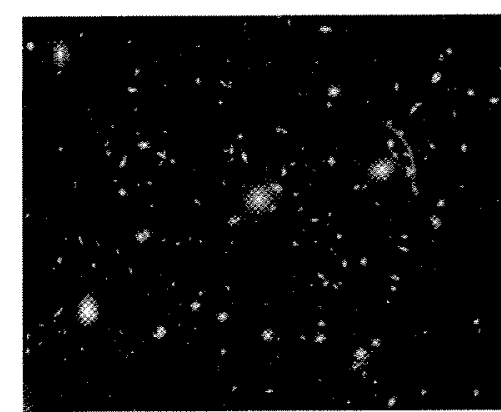

(a)

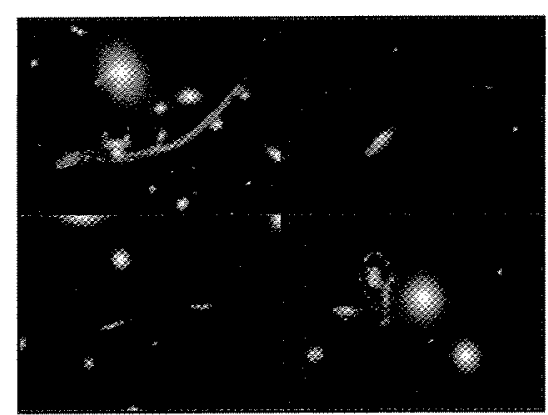

(b)

Fig. 7. (a) Restored-ACS image of Abell Cluster 370. Full-frame view shows the giant cD elliptical galaxies which dominate the cluster (center and right of center), and the "giant arc" (right of the right-most $\mathrm{cD}$ ). (b) Close-up of some of the strong-lensed features of the cluster, including the giant arc in upper left. 
in part by their DM distributions, and the reader is referred to Bradac et al. ${ }^{69,70}$ Clearly, HST has much more SL and WL work to do in pursuit of Dark Matter findings, and the future looks very promising.

\section{Conclusion}

With the addition of two new advanced instruments and the restoration of two others, HST is more scientifically powerful than ever. The three cosmological themes briefly discussed here represent a very small fraction of the total variety of science of which HST is capable, and the work cited is just the beginning of what will come in the three themes that were examined. The author draws particular attention to the recent addition of "Multi-Cycle Treasury Programs" (MCT) to the HST scientific programme. These are very large programs - each comprising at least 450 orbits of data spread over three years (cycles)--that enable large scientific gains not possible with smaller orbit allocations. The combination of MCT and the new/restored instruments should produce huge, multi-use datasets whose benefit to the community will be felt for decades to come.

It is to be noted that the emrrgence and evolution of large-scale structure is not the domain just of the HST imagers through DM lensing studies. The Cosmic Origins Spectrograph (COS) - not discussed in detail here because its science still lies ahead-will probe the cosmic web of baryonic (and dark) matter and make major discoveries fit for presentation at a future conference in this series. In summation, the next five or more years with HST look to be as or more exciting than the first twenty have been.

\section{Acknowledgments}

The author serves as NASA's Observatory Project Scientist for HST, and wishes to acknowledge his thanks to Garth Illingworth, Rychard Bowens and Adam Riess for their contribution of figures to the paper; to Zolton Levay of the Space Telescope Science Institute (STScI) for conversion of several of the images; and to Randy Kimble for a critical reading of the manuscript.

\section{References}

1. M. Niedner, in The Critical Path, 17, No. 1, NASA/GSFC Flight Projects Directorate Newsletter (2009). Article available at: http://www.nasa.gov/ mission_pages/hubble/servicing/SM4/main/HubbleScientificPinnacle.html.

2. R. Kimble, J. Mackenty, R. O'Connell and J. Townsend, Proc. SPIE 7010, 70101E (2008).

3. J. Mackenty, R. Kimble, R. O'Connell and J. Townsend, Proc. SPIE 7010, 70101F (2008).

4. M. Wong, C. Pavlovsky and K. Long et al., Wide Field Camera 3 Instrument Handbook, v. 2.0 (2010), aval. at: http:/ www.stsci.edu/hst/wfc3/documents/ handbooks/currentIHB/wfc3_cover.html

5. J. Green, E. Wilkinson and J. Morse, Proc. SPIE 5164, 17 (2003). 
6. C. Froning and J. Green, Astrophys. Space Sci. 320, 181 (2009).

7. W. Dixon et al, Cosmic Origins Spectrograph Instrument Handbook, v. 2.0 (2010), avail, at: http://www.stsci.edu/hst/cos/documents/handbooks/ current/cos_cover.html.

8. H. Ford, P. Feldman and D. Golimowski et al., Proc. SPIE 2807, 184 (1996).

9. S. Beckwith, M. Stiavelli and A. Koekemoer et al., Astron. J. 132, 1729 (2006).

10. M. Giavalisco, H. Ferguson and A. Koekemoer et al., Astrophys. J. 600, L93 (2004).

11. N. Scoville, H. Aussel and M. Brusa et al., Astrophys. J. Suppl. Ser. 172, 1 (2007).

12. N. Scoville, R. Abraham and H. Aussel et al., Astrophys. J. Suppl. Ser. 172, $38(2007)$.

13. S. Rinehart, E. Cheng and M. Sirianni et al., Proc. SPIE 7010, 70104Q (2008).

14. A. Maybhate et al., Advanced Camera for Surveys Instrument Handbook, v. 9.0 (2010), avail. at: http://www.stsciedu/hst/acs/documents/handbooks/ cycle18/cover.html.

15. B. Woodgate, R. Kimble and C. Bowers et al., Publ. Astron. Soc. Pacific 110, 1183 (1998).

16. R. Kimble, B. Woodgate and C. Bowers et al., Astrophys. J. 492, L83 (1998).

17. M. Sarzi, H-W Rix, J. Shields, G. Rudnick, L. Ho, D. McIntosh, A. Filippenko and W. Sargent, Astrophys. J. 550, 65 (2001).

18. A. Beifiori, M. Sarzi, E. Corsini, E. Bontà, A. Pizzella, L. Coccato and F. Bertola, Astrophys. J. 692, 856 (2009).

19. D. Charbonneau, T. Brown, R. Noyes and R. Gilliland, Astrophys. J. 568, 377 (2002).

20. A. Vidal-Madjar, J. Désert, A. des Etangs, G. Hébrard, G. Ballester, D. Ehrenreich, R. Ferlet, J. McConnell, M. Mayor and C. Parkinson, Astrophys. J. 604, L69 (2004).

21. S. Rinehart, J. Domber, T. Faulkner, T. Gull, R. Kimble, M. Klappenberger, D. Leckrone, M. Niedner, C. Proffitt, H. Smith and B. Woodgate, Proc. SPIE 7010, 70104R (2008).

22. C. Proffitt et al., Space Telescope Imaging Spectrograph Instrument Handbook, v. 9.0 (2010), avail. at: http://www.stsci.edu/hst/stis/documents/ handbooks/currentIHB/cover.html.

23. A. Riess, L. Macri and W. Li et al., Astrophys. J. Suppl. Ser. 183, 109 (2009).

24. A. Riess, L. Macri and S. Casertano et al., Astrophys. J. 699, 539 (2009).

25. M. Swain, G. Tinetti and G. Vasisht et al., Astrophys. J. 704, 1616 (2009).

26. A. Sandage, Astrophys. J. 331, 605 (1988).

27. W. Freedman, B. Madore and B. Gibson et al., Astrophys. J. 553, 47 (2001).

28. A. Sandage, G. Tammann, A. Saha, B. Reindl, F. Macchetto and N. Panagia, Astrophys. J. 653, 843 (2006).

29. W. Freedman, B. Madore and J. Mould et al., Nature 371, 757 (1994).

30. R. Kennicutt, W. Freedman and J. Mould, Astron. J. 110, 1476 (1995).

31. A. Saha, A. Sandage, L. Labhardt, G. Tammann, F. Macchetto and N. Panagia, Astrophys. J., 486, 1 (1997).

32. A. Riess, A. Filippenko and P. Challis et al., Astron. J. 116, 1009 (1998).

33. S. Permutter, G. Aldering and G. Goldhaber et al., Astrophys. J. 517, 565 (1999).

34. A. Riess, P. Nugent and R. Gilliland et al., Astrophys. J. 560, 49 (2001).

35. R. Knop, G. Aldering and R. Amanullah et al., Astrophys. J. 598, 102 (2003).

36. E. Komatsu, J. Dunkley and M. Nolta et al., Astrophys. J. Suppl. Ser. 180, 330 (2009).

37. J. Holtzman, J. Hester and S. Casertano et al., Publ. Astron. Soc. Pacific 107, 156 (1995).

38. A. Argon, L. Greenhill, M. Reid, J. Moran and E. Humphreys, Astrophys. J. 659 , $1040(2007)$

39. J. Braatz, M. Reid, L. Greenhill, J. Condon, K. Lo, C. Henkel, N. Gugliucci and L. 
Hao, Publ. Astron. Soc. Pactic Conference Series 395, 103 (2008).

40. L. Greenhill, P. Kondratko, J. Moran and A. Tilak, Astrophys. J. 707, 787 (2009).

41. J. Frieman, M. Turner and D. Huterer, Ann. Rev. Astron. Astrophys. 46, 385 (2008).

42. X. Fan, C. Carilli and B. Keating, Ann. Rev. Astron. Astrophys. 44, 415 (2006).

43. A. Loeb, Publ. Astron. Soc. Pacific Conference Series 395, 59 (2008).

44. S. White and M. Rees, Mon. Not. Roy. Astron. Soc. 183, 341 (1978).

45. J. Gardner, J. Mather and M. Clampin et al., Space Sci. Rev. 123, 485 (2006).

46. R. Williams, B. Blacker and M. Dickinson et al., Astron. J. 112, 1335 (1996).

47. H. Ferguson, M. Dickinson and R. Williams, Ann. Rev. Astron. Astrophys. 38, 667 $(2000)$.

48. C. Steidel, M. Pettini and D. Hamilton, Astron. J. 110, 2519 (1995).

49. R. Bouwens, R. Thompson, G. Illingworth, M. Franx, P. van Dokkum, X. Fan, M. Dickinson, D. Eisenstein and M. Rieke, Astrophys. J. 616, L79 (2004).

50. P. Oesch, R. Bouwens, G. Illingworth, C. Carollo, M. Franx, I. Labbé, D. Magee, M. Stiavelli, M. Trenti and P. van Dokkum, Astrophys. J. 709, L16 (2010).

51. R. Bouwens, G. Illingworth and P. Oesch et al., Astrophys. J. 709, L133 (2010).

52. R. McLure, J. Dunlop, M. Cirasuolo, A. Koekemoer, E. Sabbi, D. Stark, T. Targett and R. Ellis, Mon. Not. Roy. Astron. Soc. 403, 960 (2010).

53. M. Trenti, M. Stiavelli, R. Bouwens, P. Oesch, J. Shull, G. Illingworth, L. Bradley and C. Carollo, Astrophys. J. 714, L202 (2010).

54. I. Labbé, V. González and R. Bouwens et al., Astrophys. J. 708, L26 (2010).

55. R. Bouwens, G. Illingworth and I. Labbé et al., submitted to Nature (2010).

56. H. Yan, R. Windhorst, N. Hathi, S. Cohen, R. Ryan, R. O'Connell and P. McCarthy, submitted to Astrophys. J. (2010).

57. A. Réfrégier, Ann. Rev. Astron. Astrophys. 41, 645 (2003).

58. A. Newman, T. Treu, R. Ellis, D. Sand, J. Richard, P. Marshall, P. Capak and S. Miyazaki, Astrophys. J. 706, 1078 (2009).

59. A. Maizy, J. Richard, M. De Leo, R. Pelló and J. Kneib, Astron. Astrophys. 109, A105 (2010).

60. R. Bouwens, G. Illingworth, L. Bradley, H. Ford, M. Franx, W. Zheng, R. Broadhurst, D. Coe and J. Jee, Astrophys. J. 690, 1764 (2009).

61. G. Smith, H. Ebeling and M. Limousin et al., Astrophys. J. 707, L163 (2009).

62. A. Zitrin, T. Broadhurst, Y. Rephaeli and S. Sadeh, Astrophys. J. 707, L102 (2009).

63. C. Faure, J-P Kneib and G. Covone et al, Astrophys. J. Suppl. Ser. 176, 19 (2008).

64. T. Treu, Ann. Rev. Astron. Astrophys. 48, 87 (2010).

65. R. Massey, J. Rhodes and A. Leauthaud et al., Astrophys. J. Suppl. Ser. 172, 239 $(2007)$.

66. G. Soucail, B. Fort, Y. Mellier and J. Picat, Astron. Astrophys. 172, L14 (1987).

67. G. Soncail, Y. Mellier, B. Fort, F. Hammer and G. Mathez, Astron. Astrophys. 184, L7 (1987).

68. J. Richard, J. Kneib, M. Limousin, A. Edge and E. Jullo, Mon. Not. Roy. Astron. Soc. 402, L44 (2010).

69. M. Bradač, D. Clowe, A. Gonzalez, P. Marshall, W. Forman, C. Jones, M. Markevitch, S. Randall, T. Schrabback and D. Zaritsky, Astrophys. J. 652, 937 (2006).

70. M. Bradac, S. Allen, T. Tren, H. Ebeling, R. Massey, R. Morris, A. von der Linden and D. Applegate, Astrophys. J. 687, 959 (2008). 